\title{
Lung ultrasound vs. chest $X$-ray in children with suspected pneumonia confirmed by chest computed tomography: A retrospective cohort study
}

\author{
CUI YAN ${ }^{1}$, REN HUI ${ }^{2}$, ZHANG LIJUAN ${ }^{3}$ and YANG ZHOU ${ }^{1}$ \\ Departments of ${ }^{1}$ Ultrasound Diagnosis and ${ }^{2}$ Respiratory Medicine, \\ The Affiliated Hospital of Inner Mongolia Medical University, Hohot, Inner Mongolia Autonomous Region 010059; \\ ${ }^{3}$ Department of Ultrasound, Maternal and Child Health, Care Hospital of Linhe District, Bayanzhuoer, \\ Inner Mongolia Autonomous Region 015022, P.R. China
}

Received May 23, 2019; Accepted November 13, 2019

DOI: $10.3892 /$ etm.2019.8333

\begin{abstract}
The chest X-ray is routinely requested by pediatricians for children with suspected pneumonia, but has been demonstrated to be an insensitive method with relatively low accuracy. Computed tomography (CT) allows for the characterization of the consolidation in pneumonia but has a high risk of radiation exposure in children. Lung ultrasonography can identify subpleural lung consolidation in adults, but it is not accepted in routine clinical practice and is also not used for the diagnosis of children with pneumonia. The objective of the present study was to compare diagnostic parameters of lung ultrasound with chest X-ray in children with suspected pneumonia, using CT as a reference standard. Medical records of 949 children, aged $\leq 16$ years, with suspected pneumonia were reviewed. Data regarding the chest radiograph, lung ultrasound and chest CT were collected and analyzed. Beneficial score analysis for each diagnostic modality was evaluated according to the pneumonia severity index. The chest radiograph successfully detected subpleural lung consolidation and dense lung opacity. The lung ultrasound successfully detected pleural effusion and perilesional inflammatory edema. The chest $\mathrm{CT}$ successfully detected a liquefied area, enhancement and necrosis of the lungs. Compared with the chest CT, the lung ultrasound displayed 0.906 sensitivity and 0.661 accuracy, while the chest radiograph displayed 0.793 sensitivity
\end{abstract}

Correspondence to: Dr Yang Zhou, Department of Ultrasound Diagnosis, The Affiliated Hospital of Inner Mongolia Medical University, 1 Tongdao North Road, Hohot, Inner Mongolia Autonomous Region 010059, P.R. China

E-mail: albertabaldwinssr@yahoo.com

Abbreviations: CT, computed tomography; MRI, magnetic resonance imaging

Key words: air bronchogram, computed tomography, lung consolidation, pneumonia, ultrasound, X-ray and 0.559 accuracy. For a pneumonia severity index of $<3$, the chest CT displayed a good beneficial score, followed by the lung ultrasound and chest radiograph. In conclusion, lung ultrasound is a non-invasive and simple method that could be used for the diagnosis of suspected pneumonia in children.

\section{Introduction}

Pneumonia is a leading cause of disease among children in China (1). For children with suspected pneumonia, the diagnosis is confirmed by a single lateral view chest $\mathrm{X}$-ray, to decrease radiological exposure (2). Computed tomography (CT) provides a three-dimensional view, which not only makes it user-friendly, but also allows the characterization of the lung consolidation in pneumonia (3). Indeed, CT is the only imaging technique that can assess the whole lung parenchyma (3). Furthermore, CT displays the highest sensitivity and specificity for the majority of lung diseases, except for pleural effusions (3), but has a high risk of radiation exposure in children (4). Magnetic resonance imaging (MRI) has several advantages over CT, including the lack of ionizing radiation, the ability to better characterize tissues and the provision of high soft-tissue contrast (5). However, MRI is rarely the preferred choice for the evaluation of pulmonary parenchyma due to the decreased likelihood of having proton content in the lungs, as well as possible respiratory and cardiac pulsation artifacts (5). Ultrasonography is a non-radiating imaging method (6) that can identify subpleural lung consolidation in adults with pneumonia (7). Lung ultrasound is a promising adjunctive technique in patients with community-acquired pneumonia, by which radiation can be avoided (8) and secondary technical parameters (for example, a thinner chest wall) can be successfully evaluated (9). However, lung ultrasound is operator-dependent and requires skilled sonographers (10), therefore, ultrasonography is not typically used in routine clinical practice or included as a diagnostic method for pneumonia in children (11). The chest X-ray is typically requested by pediatricians in children with suspected pneumonia (12), however, this method has been suggested to be insensitive and has relatively low accuracy $(13,14)$. 
The objective of the present study was to compare diagnostic parameters of lung ultrasound with chest X-ray in children with suspected pneumonia, in a Chinese setting. In the present study, chest CT was considered as a reference standard and the pneumonia severity index was used as a level of confidence.

\section{Materials and methods}

Ethics. The protocol was approved by the Ethics Committee of the Affiliated Hospital of Inner Mongolia Medical University.

Study population. Children that were admitted to the Department of Pediatrics at the Affiliated Hospital of Inner Mongolia Medical University and the Department of Pediatrics at the Maternal and Child Health Care Hospital of Linhe District between 12 January 2018 and 28 December 2018 were evaluated for inclusion in the present study. All patients from the Maternal and Child Health Care Hospital of Linhe District were referred to the Department of Pediatrics at the Affiliated Hospital of Inner Mongolia Medical University. In total, 989 children presented with fever, dyspnea, cough, expectoration of purulent sputum, decreased breath sounds, pleuritic chest pain and/or the other clinical signs suggesting pneumonia. Among them, seven patients were aged $>16$ years, therefore, data from these patients were excluded from the present analysis. According to the Institute Guidelines, patients are considered children when aged $\leq 16$ years. Additionally, 12 children had not undergone all examinations for the diagnostic methods (ultrasound and/or CT) and 21 children had confirmed pneumonia and were on antibiotic treatment, therefore these data were also excluded from the analysis. The flowchart of analysis is presented in Fig. 1.

Data collection. Data regarding clinicopathological conditions, demographical characteristics, clinical examinations, the chest X-ray, transthoracic sonography and the chest CT of 949 children were collected from the institutional records. Data were collected electronically and anonymized.

Clinical examinations. Within $24 \mathrm{~h}$ of admission, blood samples were collected and sent to a laboratory at the Affiliated Hospital of Inner Mongolia Medical University for pathological examination, and data regarding blood chemistry were evaluated.

Chest radiograph. Children in a seated position received frontal and lateral view chest X-rays using DuraDiagnost (Koninklijke Philips N.V.). Image interpretation was performed by three pediatric radiologists with $\geq 5$ years' experience, who were blinded to the results of the clinical examination, at each institute.

Lung ultrasound. Transthoracic sonography was performed by sonographers ( $\geq 3$ years of experience) at each institute, on patients in a seated or supine position; using EPIQ Elite (Koninklijke Philips N.V.) in B-mode connected to $7.5 \mathrm{MHz}$ linear probes (Koninklijke Philips N.V.). Scans included two rib spaces per lung field for the anterior, mild axillary and posterior fields, totaling to six scanning windows per hemithorax (15). Image interpretation was performed by sonographers with $\geq 5$ years' experience, who were blinded to the results of clinical examinations and chest X-rays, in consultation with pulmonologists ( $\geq 3$ years' experience) at each institute. Transthoracic ultrasound was performed in real time.

Chest CT. Chest CT scans were performed on patients from the level of the thoracic inlet to the diaphragm using an eight-sliced scanner (Koninklijke Philips N.V.) at 100 kV, 25 mAs, 0.14 volume, $0.5 \mathrm{sec}$ rotation and $2 \times 10$ collimation. CT scans were performed by radiologists $(\geq 5$ years' experience; blinded regarding results of clinical examinations, chest X-rays and lung ultrasound) at each institute. Images were analyzed by radiologists in consultation with pulmonologists ( $\geq 3$ years' experience) at each institute (16). When images were reviewed, $\geq 3$ independent interpretations of the results were performed. Pleural effusion, perilesional inflammatory edema and lung consolidations were considered as pneumonia. True, false or inconclusive results of lung ultrasound and chest radiography were decided based on the results obtained from chest CT.

Beneficial analysis. Beneficial score analysis was performed to analyze an area to detect positive disease (pneumonia) by imaging methods, or for the evaluation of imaging modality performance. Beneficial score analysis was evaluated according to the pneumonia severity index for each diagnostic modality, using the following equation (17): (true positive pneumonia/949)-[(false positive pneumonia/949) $\mathrm{x}$ ([5-LC]/LC)], where true positive pneumonia means that pneumonia was detected by imaging modalities as well as by the chest $\mathrm{CT}$; false positive means that pneumonia was detected by imaging modalities but not detected by the chest CT; LC is the level of confidence above which children were put on antibiotics, which was determined by the pneumonia severity index, a 0 to 5 scale, according to Institutional Guidelines for Pediatric Pneumonia. The scale was defined as follows: 0 , absent (no clinical signs and symptoms); 1 , mild pneumonia (clinical signs and symptoms but no hospitalization required); 2 , mild to moderate pneumonia (hospitalization but no intensive care admission); 3 , moderate pneumonia (intensive care admission but no mechanical ventilation required); 4 , moderate to severe pneumonia (mechanical ventilation but no death); and 5, severe pneumonia (shock or death). The ratio of true positive pneumonia detected by each modality to true positive pneumonia detected by chest CT was considered sensitivity. The ratio of true negative pneumonia detected by each modality to true negative pneumonia detected by chest CT was accuracy.

Statistical analysis. Categorical variables are presented as a number and percentage. Continuous variables are presented as the mean \pm SD. SPSS software (version 24.0; IBM Corporation) was used for statistical analysis. Categorical data were analyzed by the $\chi^{2}$ test. A P $<0.05$ was considered to indicate a significant difference.

\section{Results}

Clinical manifestations. Dyspnea and cough were the most commonly reported symptoms among the patients, followed 


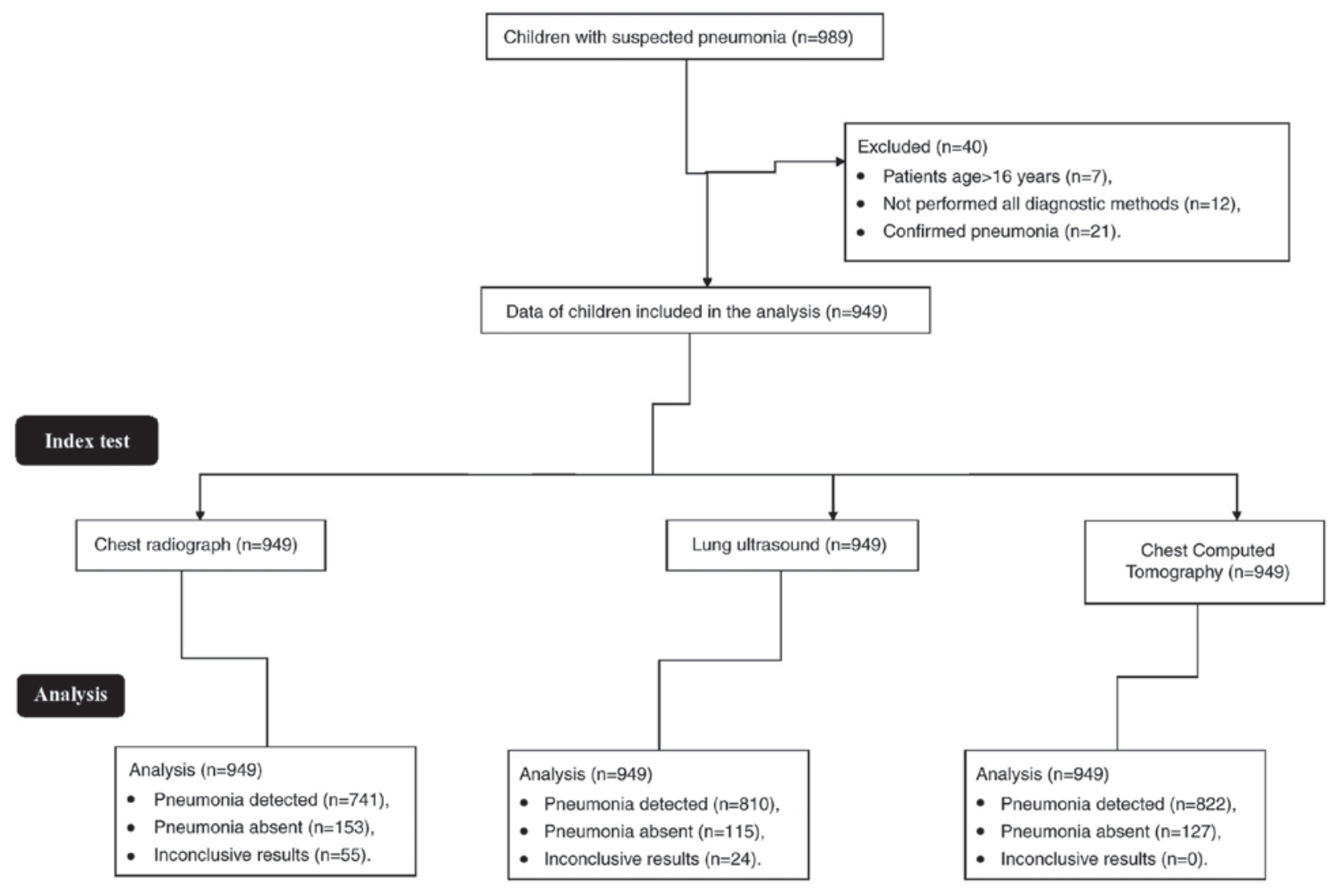

Figure 1. Flowchart of data analysis.

by expectoration of purulent sputum, fever and pleuritic chest pain. Further demographical characteristics of the patients are presented in Table I.

Laboratory tests. Children displayed a higher erythrocyte sedimentation rate than normal. A total of $171(18 \%)$ patients displayed severe hypoxemia and were subsequently admitted into the Emergency Department. The results of further laboratory tests are presented in Table II.

Chest radiograph. The chest X-ray successfully detected subpleural lung consolidation (Fig. 2) and dense opacity in the lungs, but was unable to identify whether the lung had undergone suppurative, necrotic change or was congested (Fig. 3).

Lung ultrasound. Transthoracic sonography successfully detected a minimal pleural effusion and perilesional inflammatory edema (Fig. 4). However, a lung consolidation with an air bronchogram, as well as the consolidations that did not reach the pleura were missed. Lung ultrasound was also not able to define the hyperechoic spots present in the consolidations as an air bronchogram.

Chest $C T$. The chest CT successfully detected liquefied areas, inflammation and necrosis of the lungs (Fig. 5).

Diagnostic parameters. Compared with chest CT, lung ultrasound displayed 0.906 sensitivity and 0.661 accuracy, while chest radiograph displayed 0.793 sensitivity and 0.559

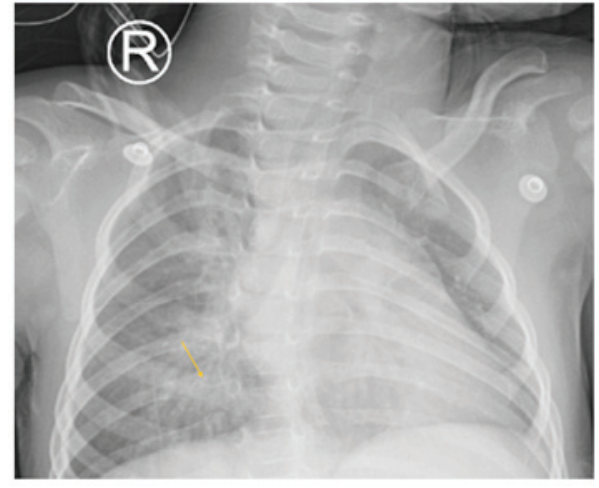

Figure 2. Lateral view chest X-ray of a 6-year old boy with productive cough. Yellow arrow indicates subpleural lower right lung consolidation.

accuracy. Further diagnostic parameters of imaging modalities are presented in Table III.

Beneficial analysis. All diagnostic modalities displayed considerable beneficial scores when the pneumonia severity index was $\geq 3$. For pneumonia severity indexes $<3$, the chest CT provided the highest beneficial score, followed by lung ultrasound and chest radiography, respectively (Fig. 6).

\section{Discussion}

The present study reported that chest X-ray was unable to identify necrosis and congestion of the lungs in pediatric patients with suspected pneumonia. The air bronchogram 
Table I. Clinicopathological and demographical characteristics.

\begin{tabular}{lc}
\hline Characteristic & Patient population \\
\hline Age (years) & 2 \\
Minimum & 16 \\
Maximum & $12.45 \pm 3.12$ \\
Mean \pm SD & \\
Sex & $446(47)$ \\
Male & $503(53)$ \\
Female & \\
Ethnicity & $871(92)$ \\
Han Chinese & $62(2)$ \\
Mongolian & $16(2)$ \\
Tibetan & $361(38)$ \\
Fever & $711(75)$ \\
Dyspnea & $778(82)$ \\
Cough & $3,445(47)$ \\
Expectoration of purulent sputum & $145(15)$ \\
Decreased breath sounds & $345(36)$ \\
Pleuritic chest pain & $281(30)$ \\
Chills & $157(17)$ \\
Headache &
\end{tabular}

Constant variables are presented as number (percentage). Continuous variables are presented as mean $\pm \mathrm{SD}$.

Table II. Biological characteristics.

\begin{tabular}{lc}
\hline Characteristic & Patient population \\
\hline Erythrocyte sedimentation rate $(\mathrm{mm} / \mathrm{h})^{\mathrm{a}}$ & $20.12 \pm 7.22$ \\
White blood cell/dl & $12,545.00 \pm 3,415.00$ \\
Procalcitonin $(\mu \mathrm{g} / \mathrm{l})$ & $2.11 \pm 0.54$ \\
Serum reactive protein $(\mathrm{mg} / \mathrm{l})$ & $111.85 \pm 12.17$ \\
Urine area $>11 \mathrm{mM} / \mathrm{l}$ & $141(15)$ \\
Blood $\mathrm{pH}<7.35$ & $10(1)$ \\
Severe hypoxemia $($ partial pressure of & $171(18)$ \\
oxygen in blood $<60 \mathrm{mmHg})$ & \\
\hline
\end{tabular}

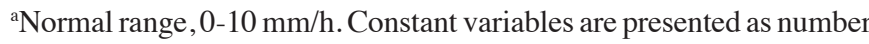
(percentage). Continuous variables are presented as mean $\pm \mathrm{SD}$.

was only identified by chest CT. The results from the present study were consistent with prospective studies $(16,18)$ and retrospective reviews on adult patients $(13,14)$. However, the results obtained were not in line with a prospective observational cohort study on lung ultrasound in children and young adults (19). Chest CT is considered as 'gold standard' in the detection of pneumonia (20), but cannot be used routinely in children because children are radiosensitive and display a greater risk associated with radiation than adults (3). Therefore, there is a need for an alternative diagnostic modality for the detection of pneumonia in children, with a reduced risk of radiation exposure.

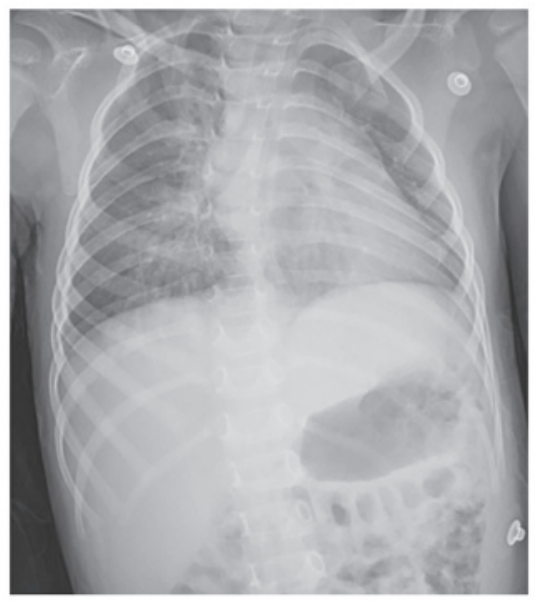

Figure 3. Lateral view chest X-ray of a 5-year old girl with productive cough and fever, in the supine position. The chest X-ray image did not identify pneumonia that was later detected by chest $\mathrm{CT}$, which displayed a low-density area in the lungs, and by lung ultrasound, which identified perilesional inflammatory edema.

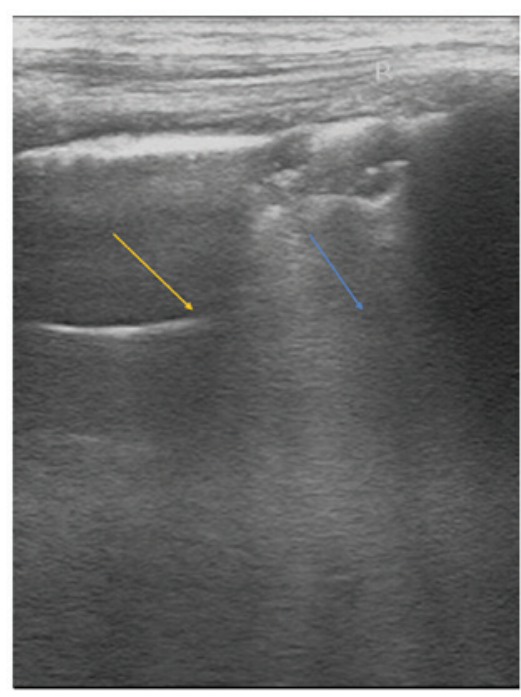

Figure 4. Lung ultrasound in the supine position of a 5-year girl old with a productive cough and fever. The yellow arrow indicates pleural effusion of the lung. The blue arrow indicates perilesional inflammatory edema.

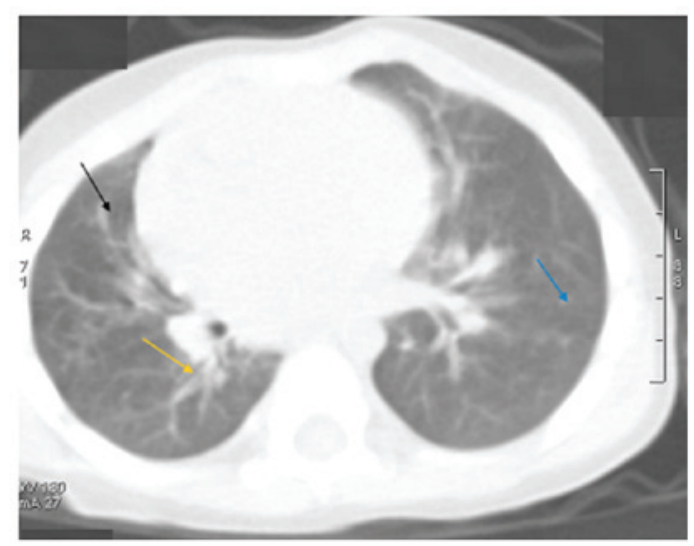

Figure 5. Axial view of the chest CT of a 7-year old boy with a productive cough and fever. The black arrow indicates poorly enhancing or liquefied areas of the lung or low-density areas of the lungs. The yellow arrow indicates an enhancement of the lungs. The blue arrow indicates necrosis of the lungs. 
Table III. Diagnostic parameters of imaging modalities.

\begin{tabular}{|c|c|c|c|c|c|}
\hline \multirow[b]{3}{*}{ Parameter } & \multicolumn{5}{|c|}{ Imaging modality } \\
\hline & \multirow{2}{*}{$\frac{\text { Chest computed tomography }}{\text { Patient population }}$} & \multicolumn{2}{|c|}{ Lung ultrasound } & \multicolumn{2}{|c|}{ Chest radiograph } \\
\hline & & Patient population & P-value & Patient population & P-value \\
\hline True positive pneumonia & $822(87)$ & 745 (79) & $<0.0001$ & $652(69)$ & $<0.0001$ \\
\hline True negative pneumonia & $127(13)$ & $84(9)$ & 0.0020 & $71(7)$ & $<0.0001$ \\
\hline False positive pneumonia & $0(0)$ & $65(7)$ & $<0.0001$ & $89(9)$ & $<0.0001$ \\
\hline False negative pneumonia & $0(0)$ & $31(3)$ & $<0.0001$ & $82(9)$ & $<0.0001$ \\
\hline Inconclusive results & $0(0)$ & $24(2)$ & $<0.0001$ & $55(6)$ & $<0.0001$ \\
\hline Sensitivity & 1 & 0.906 & $<0.0001$ & 0.793 & $<0.0001$ \\
\hline Accuracy & 1 & 0.661 & $<0.0001$ & 0.559 & $<0.0001$ \\
\hline
\end{tabular}

Constant variables are presented as number (percentage). Continuous variables are presented as the mean. Variables were analyzed by the $\chi^{2}$ test. Image interpretation of chest radiograph was performed by pediatric radiologists at each institute. Image interpretation of lung ultrasound was performed by sonographers at each institute. Image interpretation of the chest computed tomography was performed by radiologists at each institute. All radiographers and sonographers had $\geq 5$ years' experience. When images were reviewed, $\geq 3$ independent interpretations of the results were performed. Pleural effusion, perilesional inflammatory edema and lung consolidations were considered as pneumonia for images. True, false or inconclusive results by lung ultrasound and chest radiograph were decided on the results obtained from chest computed tomography. $\mathrm{P}<0.05$ was considered to indicate a statistically significant difference, compared with chest computed tomography.

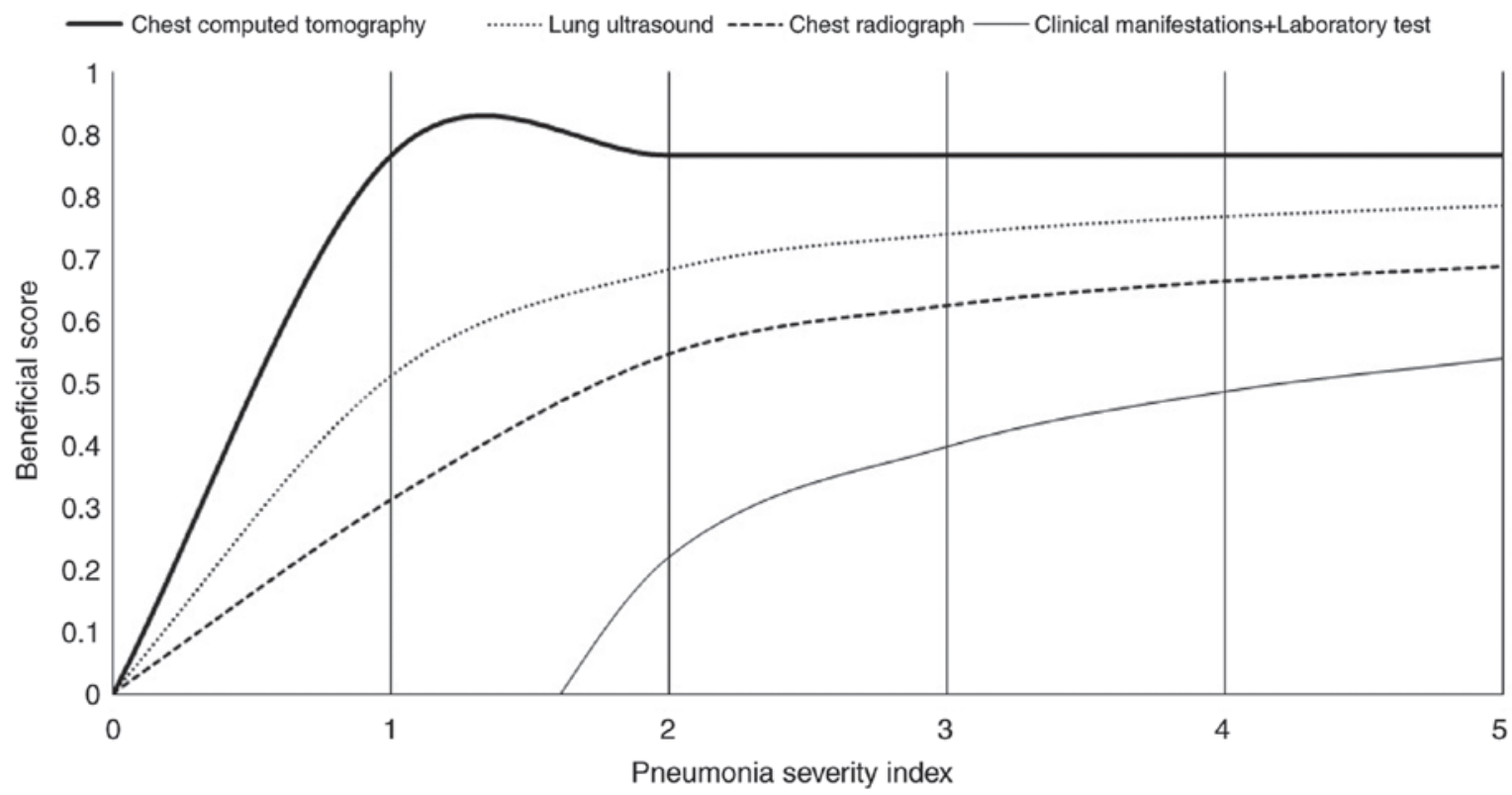

Figure 6. Beneficial score analysis of the different diagnostic modalities across pneumonia severity indexes. Image interpretation of chest X-rays was performed by pediatric radiologists at each institute. Image interpretation of lung ultrasounds was performed by sonographers at each institute. Image interpretation of chest CTs was performed by radiologists at each institute. All radiologists and sonographers had $\geq 5$ years of experience. 0 , no pneumonia; 1 , mild pneumonia; 2 , mild to moderate pneumonia; 3 , moderate pneumonia; 4 , moderate to severe pneumonia; 5 , severe pneumonia.

In the present study, for the detection of pneumonia, lung ultrasound displayed 0.906 sensitivity and 0.661 specificity, while chest X-ray displayed 0.793 sensitivity and 0.559 specificity, compared with chest CT. These results were consistent with results reported by a number of prospective diagnostic accuracy studies $(4,11,21)$. The chest X-ray is routinely recommended as an important diagnostic modality for pneumonia because it can predict pneumonia without clinical signs (9). Likewise, lung ultrasound is successful in the differential diagnosis of atelectasis and lung consolidation (22), because fluid or solid material that accumulates in the lung can be easily visualized by a transthoracic sonograph (10). Additionally, children have a smaller lung mass and thinner chest walls compared to adults, which makes lung ultrasound ideal for the diagnosis of pneumonia in children (23). Even a low dose $(<0.2 \mathrm{mGy})$ of chest X-ray is harmful to children (20). Furthermore, chest X-ray does not display $100 \%$ specificity or accuracy and also has issues of intra- and inter-observer disagreements (23). Alternatively, the results of the current study indicated that lung ultrasound was a safe, reliable and 
superior diagnostic method compared with chest X-ray for the diagnosis of pneumonia in children.

Lung ultrasound displayed 65 (7\%) false-positive results, whereas chest X-ray resulted in 89 (9\%) false-positive results $(\mathrm{P}=0.053)$ for the diagnosis of pneumonia. These results were consistent with a prospective diagnostic accuracy study (4). However, these results were not in line with a prospective observational cohort study investigating the use of ultrasound for children and young adults (19). Chest $\mathrm{X}$-ray has an issue resulting in the blending of various tissue images, whereas lung ultrasound allows dynamic analysis of each intercostal space during breathing (4). Ultrasonography of the left lower chest is problematic as artifacts are caused by the interaction between the ultrasound beam and even a small amount of fluid and air. This implies that pleural effusion enhances artifact generation (19). In the present study lung ultrasound resulted in fewer false-negative results than chest $\mathrm{X}$-ray ( 31 vs. $82 ; \mathrm{P}<0.0001$ ). The results from the present study were consistent with a number of prospective diagnostic accuracy studies $(7,19)$. X-ray has poor sensitivity for small subpleural consolidations (4) and can only detect lung consolidations that are $\geq 1.5 \mathrm{~cm}$ (19). The results of the current study reported that false predictive values of lung ultrasound were lower than those for chest X-ray and could be eliminated by integrating cardiac and abdominal scans of the lung, through the diaphragm, with the intercostal approach.

In the present study, for pneumonia severity indexes $<3$, lung ultrasound displayed a higher beneficial score than chest $\mathrm{X}$-ray, indicating that lung ultrasound may aid in the clinical decision-making process regarding putting children on antibiotics in the early stages of pneumonia, when symptoms are not severe.

There were several limitations of the present study, for example, the study was a retrospective study and lacked a prospective trial. The present study performed chest CT to confirm pneumonia, but generally chest CT is not preferred as a diagnostic module in children for safety reasons. Typically, there is less inter-and intra-operator reproducibility for lung ultrasound than chest X-ray (16). The amount of experience each sonographer has can also impact the accuracy of the ultrasound findings (9). Furthermore, lung ultrasound has fewer accuracies in identifying lung abscesses than chest CT (16). Combining lung ultrasound and chest X-ray has been reported to display results consistent with chest CT (16), however, the present study did not include such analysis. Additionally, a large randomized trial is required to assess the combination of lung ultrasound and chest X-ray in the diagnosis of pneumonia in children.

In conclusion, lung ultrasound is a non-invasive and simple method for the diagnosis of suspected pneumonia in children. Unlike chest X-ray and CT, lung ultrasound is an easy method to perform in children at the bedside without ionizing radiation exposure. The present study recommended lung ultrasound for the diagnosis of pneumonia instead of using chest radiography or CT.

\section{Acknowledgements}

Not applicable.

\section{Funding}

No funding was received.

\section{Availability of data and materials}

The datasets used and/or analyzed during the present study are available from the corresponding author on reasonable request.

\section{Authors' contributions}

All authors read and approved the manuscript for publication. CY was the project administrator and contributed to the conceptualization, software and the literature review. RH contributed to formal analysis, validation, resources and the literature review. ZL contributed to the resources, data curation, investigation and the literature review. YZ contributed to the software, formal analysis and literature review of the study. $\mathrm{YZ}$ also drafted, reviewed and edited the manuscript for intellectual content. Authors agree to be accountable for all aspects of work ensuring integrity and accuracy.

\section{Ethics approval and consent to participate}

The present study was approved by the Ethics Committee of the Affiliated Hospital of Inner Mongolia Medical University. The present study complied with the 2008 Declaration of Helsinki. Written informed consent was provided by the parents of each of the pediatric patients in the present study.

\section{Patient consent for publication}

Written informed consent for the publication of the present study was provided by the parents of each of the pediatric patient, including the publication of personal data and images.

\section{Competing interests}

The authors declare that they have no competing interests.

\section{References}

1. Ning G, Wang X, Wu D, Yin Z, Li Y, Wang H and Yang W: The etiology of community-acquired pneumonia among children under 5 years of age in mainland China, 2001-2015: A systematic review. Hum Vaccin Immunother 13: 2742-2750, 2017.

2. Harris M, Clark J, Coote N, Fletcher P, Harnden A, McKean M and Thomson A; British Thoracic Society Standards of Care Committee: British Thoracic Society guidelines for the management of community acquired pneumonia in children: Update 2011. Thorax 66 (Suppl 2): iil-ii23, 2011.

3. Brenner DJ and Hall EJ: Computed tomography-an increasing source of radiation exposure. N Engl J Med 357: 2277-2284, 2007.

4. Reali F, Sferrazza Papa GF, Carlucci P, Fracasso P, Di Marco F, Mandelli M, Soldi S, Riva E and Centanni S: Can lung ultrasound replace chest radiography for the diagnosis of pneumonia in hospitalized children? Respiration 88: 112-115, 2014.

5. Ekinci A, Yucel Ucarkus T, Okur A, Öztürk M and Doğan S: MRI of pneumonia in immunocompromised patients: Comparison with CT. Diagn Interv Radiol 23: 22-28, 2017.

6. Reissig A and Kroegel C: Sonographic diagnosis and follow-up of pneumonia: A prospective study. Respiration 74: 537-547, 2007. 
7. Reissig A, Copetti R, Mathis G, Mempel C, Schuler A, Zechner P, Aliberti S, Neumann R, Kroegel C and Hoyer H: Lung ultrasound in the diagnosis and follow-up of community-acquired pneumonia: A prospective, multicenter, diagnostic accuracy study. Chest 142: 965-972, 2012.

8. Medford ARL: Chest ultrasonography as a replacement for chest radiography for community-acquired pneumonia. Chest 143 : 877-878, 2013.

9. Copetti R and Cattarossi L: Ultrasound diagnosis of pneumonia in children. Radiol Med 113: 190-198, 2008 (In English, Italian).

10. Roić G: Lung ultrasound in the diagnosis of pediatric pneumonia: Are we ready for routine use? Acta Med Acad 45: 82-83, 2016.

11. Caiulo VA, Gargani L, Caiulo S, Fisicaro A, Moramarco F, Latini G, Picano E and Mele G: Lung ultrasound characteristics of community-acquired pneumonia in hospitalized children. Pediatr Pulmonol 48: 280-287, 2013.

12. Caiulo VA, Gargani L, Caiulo S, Fisicaro A, Moramarco F, Latini G and Picano E: Sensitivity and feasibility of lung ultrasound in bronchiolitis-reply to the correspondence letter by Catalano. Eur J Pediatr 173: 407-408, 2014.

13. Hayden GE and Wrenn KW: Chest radiograph vs. computed tomography scan in the evaluation for pneumonia. J Emerg Med 36: 266-270, 2009.

14. Self WH, Courtney DM, McNaughton CD, Wunderink RG and Kline JA: High discordance of chest X-ray and computed tomography for detection of pulmonary opacities in ED patients: Implications for diagnosing pneumonia. Am J Emerg Med 31: 401-405, 2013.

15. Caiulo VA, Gargani L, Caiulo S, Fisicaro A, Moramarco F, Latini G and Picano E: Lung ultrasound in bronchiolitis: Comparison with chest X-ray. Eur J Pediatr 170: 1427-1433, 2011.
16. Hajalioghli P, Nemati M, Dinparast SL and Fouladi DF: Can chest computed tomography be replaced by lung ultrasonography with or without plain chest radiography in pediatric pneumonia? J Thorac Imaging 31: 247-252, 2016.

17. Fitzgerald M, Saville BR and Lewis RJ: Decision curve analysis. JAMA 13: 409-410, 2015.

18. Claessens YE, Debray MP, Tubach F, Brun AL, Rammaert B, Hausfater P, Naccache JM, Ray P, Choquet C, Carette MF, et al: Early chest computed tomography scan to assist diagnosis and guide treatment decision for suspected community-acquired pneumonia. Am J Respir Crit Care Med 192: 974-982, 2015.

19. Shah VP, Tunik MG and Tsung JW: Prospective evaluation of point-of-care ultrasonography for the diagnosis of pneumonia in children and young adults. JAMA Pediatr 167: 119-125, 2013.

20. Caiulo VA, Gargani L, Caiulo S, Fisicaro A, Moramarco F, Latini G, Picano E and Mele G: The role of ultrasound in community-acquired pneumonia. Pediatr Pulmonol 48: 1043-1044, 2013.

21. Cortellaro F, Colombo S, Coen D and Duca PG: Lung ultrasound is an accurate diagnostic tool for the diagnosis of pneumonia in the emergency department. Emerg Med J 29: 19-23, 2012.

22. Lichtenstein D, Mezière G and Seitz J: The dynamic air bronchogram. A lung ultrasound sign of alveolar consolidation ruling out atelectasis. Chest 135: 1421-1425, 2009.

23. Caiulo VA, Gargani L, Caiulo S, Fisicaro A, Moramarco F, Latini G, Picano E and Mele G: Response to lung ultrasound as an additional imaging tool for the evaluation of pneumonia. Pediatr Pulmonol 49: 619-620, 2014.

This work is licensed under a Creative Commons Attribution-NonCommercial-NoDerivatives 4.0 International (CC BY-NC-ND 4.0) License. 